\title{
Interaction between Plasma Protein and Microphase Separated Structure of Copolymers
}

\author{
Teruo OKano, ${ }^{*}$ Shoji Nishiyama, ${ }^{*}$ Isao Shinohara, ${ }^{*}$ \\ Toshihiro AKaIKe, ** and Yasuhisa SAKURAI** \\ *Department of Polymer Chemistry, Waseda University, Tokyo 160, Japan. \\ **Department of Surgical Science, The Heart Institute of Japan, Tokyo \\ Women's Medical College, Tokyo 162, Japan.
}

(Received October 11, 1977)

\begin{abstract}
Bovine serum albumin and $\gamma$-globulin were adsorbed, respectively, on films of ABA-type block copolymers and blendmers composed of 2-hydroxyethyl methacrylate (HEMA) and styrene in a mixture of protein and $0.2 \mathrm{M}$ of phosphate buffer solution (pH 7.4) under the condition of $1 / 50$ physiological concentration of the protein at $37^{\circ} \mathrm{C}$. The morphologies and dimensions of the microphase separated structure of the block copolymers and blendmers containing hydrophilic domains of HEMA molecules and hydrophobic domains of styrene molecules were studied by electron microscopy with or without osmium tetroxide fixation. In the adsorption of plasma protein, either both the adsorbed plasma protein and the hydrophilic domains or only the adsorbed plasma proteins could be stained selectively with osmium tetroxide to control the fixation time. Therefore the areas of plasma protein adsorbed on the phase of microseparated structure were evaluated by comparing the micrographs with and without the adsorbed plasma proteins. These results show that bovine serum albumin is adsorbed on the hydrophilic domains escaping from the hydrophobic domains, and on the contrary that $\gamma$-globulin is selectively adsorbed on the hydrophobic domains.
\end{abstract}

KEY WORDS Serum Albumin / $\gamma$-Globulin / Block Copolymer / 2 Hydroxyethyl Methacrylate / Styrene / Microphase Separated Structure / Adsorption /

When a foreign surface is in contact with blood, the surface is covered with plasma protein immediately. The protein layer on the surface affects platelet adhesion and aggregation. For example, adsorbed fibrinogen and $\gamma$-globulin are known to induce platelet aggregation, while adsorbed serum albumin does not induce platelet aggregation. ${ }^{1}$ Therefore, it is important to clarify the polymerprotein interaction on the surface in order to design antithrombogenic polymers.

It has been reported that the heterogeneity of the synthetic polymer surface, i.e., the microphase separated structure, plays an important role in blood compatibility. ${ }^{2}$ In practice, normal vascular endothelium, which is the ideal nonthrombogenic, is considered to have a microphase separated structure composed of hydrophilic and hydrophobic microdomains. $^{3}$

Block copolymers composed of the hydrophilic monomer 2-hydroxyethyl methacrylate (HEMA) and the hydrophobic monomer styrene were synthesized ${ }^{4}$ to study the effect of their microphase separated structure on blood compatibility. The interaction between the surface which has a hydrophilic-hydrophobic microphase separated structure and plasma protein was investigated by means of a new method of electron microscopy. The difference in the adsorption of serum albumin and $\gamma$-globulin on the phase of the microstructure was discussed from the standpoint of the hydrophilicity and hydrophobicity of the microphases of block copolymers and blendmers.

\section{EXPERIMENTAL}

\section{Material}

ABA-type block copolymers of HEMA (A) and styrene (B) were synthesized using telechelic oligo- 
Table I. HEMA—styrene block copolymers and blendmers

\begin{tabular}{lccc}
\hline \multirow{2}{*}{ Sample } & $\begin{array}{c}\text { HEMA } \\
\text { mole } \\
\text { fraction }\end{array}$ & \multicolumn{2}{c}{ Molecular weight } \\
\cline { 3 - 4 } & HEMA chain & St chain \\
\hline Block copolymer & 0.347 & 2420 & 7100 \\
Block copolymer & 0.608 & 10400 & 10500 \\
Block copolymer & 0.884 & 10400 & 2100 \\
Blendmer & 0.600 & 2000 & 4000 \\
Blendmer & 0.800 & 2000 & 4000 \\
\hline
\end{tabular}

mers as prepolymers by the method described in a previous paper. ${ }^{4}$ HEMA-styrene blendmers were prepared by mixing oligo-HEMA and oligo-styrene as described previously. ${ }^{5}$ Table I shows the block copolymers and the blendmers used as samples for the analysis of the adsorption of plasma protein. The microstructures of the block copolymers were observed by an electron microscope (Hitachi HU-11 Type) after staining by exposing them to the vapor of osmium tetroxide (1-wt \% aqueous solution) for $20 \mathrm{hr}$. The microstructures of blendmers were observed without staining.

\section{Analysis of Adsorbed Plasma Protein}

Bovine serum albumin and bovine $\gamma$-globulin were dissolved in $0.2 \mathrm{M}$ of phosphate buffer solution of $\mathrm{pH}$ 7.4. The concentration of the protein solution was $1 / 50$ of physiological concentration, i.e., $1 / 50 \times 4.5 \mathrm{~g} / \mathrm{d} l$ of serum albumin and $1 / 50 \times$ $1.6 \mathrm{~g} / \mathrm{d} l$ of $\gamma$-globulin. $N, N$-Dimethylformamide (DMF) solutions of the polymer were made to be cast on a carbon-coated copper grid and thin films were prepared by evaporating DMF at $40^{\circ} \mathrm{C}$ and then by drying under reduced pressure. The films were immersed in the protein solution for $30 \mathrm{sec}$. After the rinse was repeated 3 times, the samples were treated with formalin phosphate buffer solution ( $\mathrm{pH}$ 7.4) for $2 \mathrm{hr}$. The samples were dried under reduced pressure and then were stained by osmium tetroxide; exposure time for $6 \mathrm{hr}$ blendmer systems and $20 \mathrm{hr}$ for block copolymer systems. After the samples were dried under reduced pressure, their microstructures were observed by an electron microscope.

\section{RESULTS AND DISCUSSION}

\section{Adsorption of Plasma Protein on the Surface of HEMA-Styrene Blendmers}

Figure 1 shows the electron micrographs of a blendmer containing $0.6-\mathrm{mol}$ fraction of HEMA (Figure 1A) and of surface after adsorption of bovine serum albumin (Figure 1B) and $\gamma$-globulin (Figure 1C). In the case of the HEMA-styrene blendmer system, hydrophobic domains consisting of styrene molecules could be observed by the electron microscope as black circles without stain-

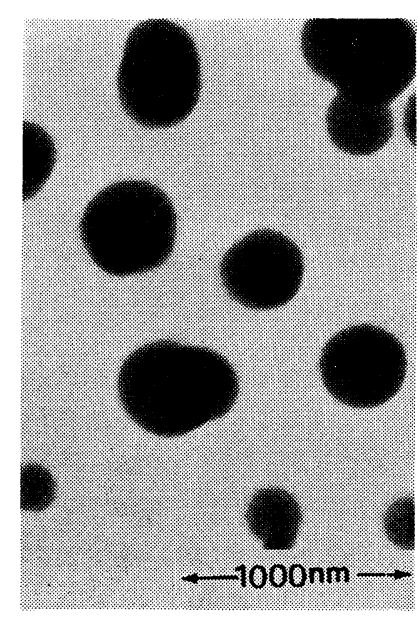

A

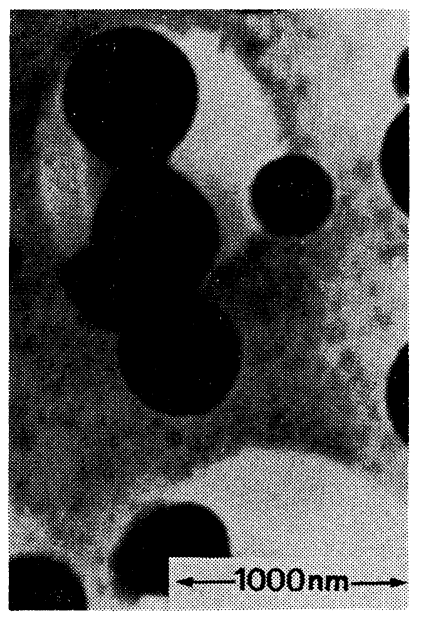

B

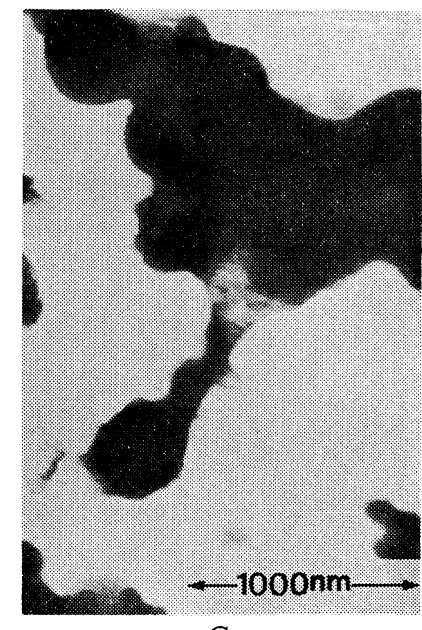

$\mathrm{C}$

Figure 1. Adsorption of plasma protein on the surface of HEMA-styrene blendmer containing 0.6-mol fraction of HEMA: (A) the surface of the blendmer; (B) the surface after adsorbing bovine serum albumin, stained with osmium tetroxide; $(\mathrm{C})$ the surface after adsorbing bovine $\gamma$-globulin, stained with osmium tetroxide. 


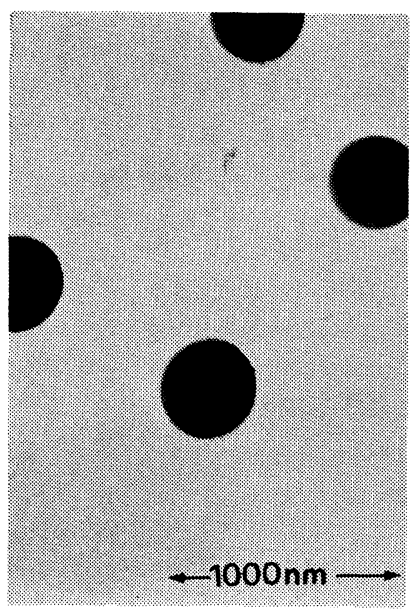

A

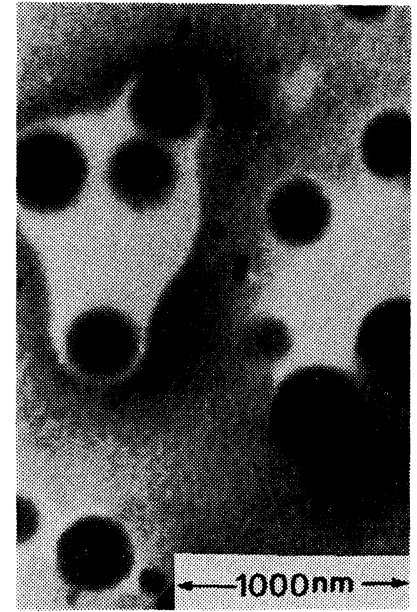

B

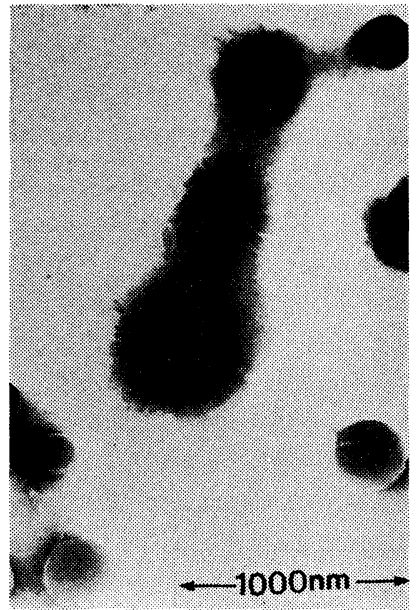

$\mathrm{C}$

Figure 2. Adsorption of plasma protein on the surface of HEMA-styrene blendmer containing 0.8-mol fraction of HEMA: (A) the surface of the blendmer; (B) the surface after adsorbing bovine serum albumin, stained with osmium tetroxide; (C) the surface after adsorbing bovine $\gamma$-globulin, stained with osmium tetroxide.

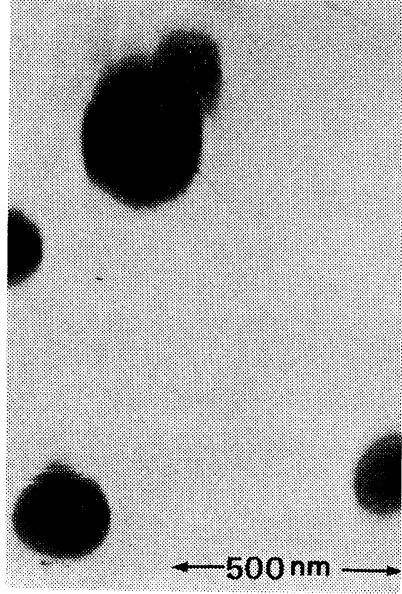

A

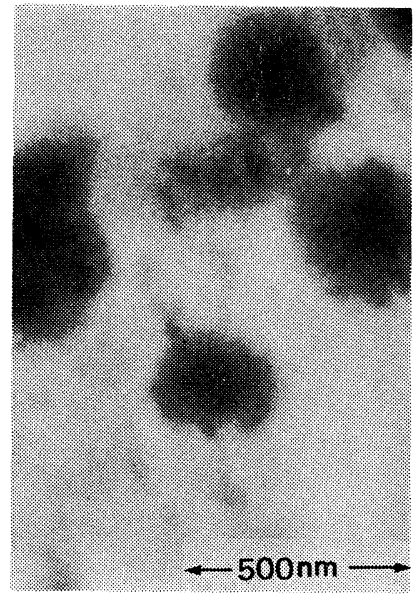

B

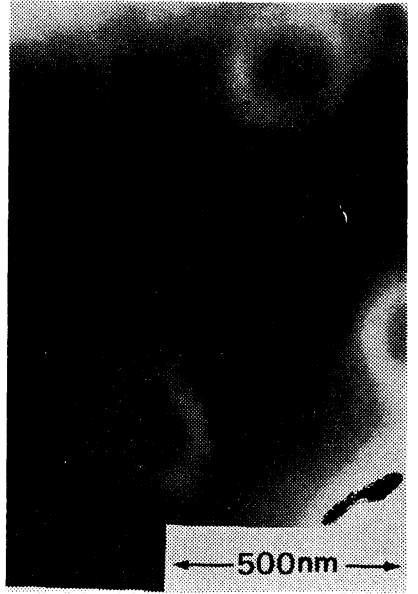

$\mathrm{C}$

Figure 3. Adsorption of plasma protein on the surface of HEMA-styrene ABA-type block copolymer containing $0.347-\mathrm{mol}$ fraction of HEMA: (A) the surface of the block copolymer stained with osmium tetroxide; (B) the surface after adsorbing bovine serum albumin, stained with osmium tetroxide; (C) the surface after adsorbing $\gamma$-globulin, stained with osmium tetroxide.

ing because the oligo-styrene molecules aggregated strongly. ${ }^{5}$ The adsorbed plasma proteins were observed as dark areas after staining by osmium tetroxide. Although both proteins and HEMA molecules are stained by osmium tetroxide, the rate of staining of proteins is more rapid than that of HEMA molecules. So the plasma proteins were selectively stained by a short time exposure to osmium tetroxide. Figure 1 indicates that serum albumin was adsorbed on the hydrophilic domain composed of HEMA and avoided the hydrophobic domain, and on the contrary that $\gamma$-globulin was selectively adsorbed on the hydrophobic domain.

The micrographs of the adsorbed plasma proteins 
on the blendmer containing 0.8 -mol fraction of HEMA are shown in Figure 2. A similar pattern of selective adsorptions appeared with respect to both serum albumin and $\gamma$-globulin.

Adsorption of Plasma Protein on the Surface of HEMA-Styrene Block Copolymers

The microstructure of ABA-type block copolymers of HEMA and styrene changed depending upon the composition of the copolymers. Thus HEMA - styrene block copolymers are appropriate materials for investigating the interaction between the surface which has a microphase separated structure and plasma protein.

Figure 3 shows the micrographs of the block copolymer containing 0.347 -mol fraction of HEMA and its surface after adsorption of serum albumin and $\gamma$-globulin. In the polymer containing 0.347mol fraction of HEMA, the hydrophilic domains were observed as isolated island spots in the sea of the hydrophobic region. The protein adsorbed sample was sufficiently stained so that both HEMA molecules and the plasma proteins were stained. Thus both regions were observed as dark areas. The areas of the adsorbed plasma proteins could be discriminated by comparing the micrographs with and without the adsorbed proteins. The adsorption of serum albumin on the surface of the block copolymer appeared to be specific to the hydro- philic domains. On the contrary, $\gamma$-globulin was the block copolymer containing 0.608 -mol fraction of HEMA are shown in Figure 4. It is apparent that the hydrophilic and the hydrophobic domains are arranged in lamellae, as shown in Figure 4A. In the albumin adsorbed sample, the white area of hydrophobic domains remained widely in comparison with the system of adsorbed $\gamma$-globulin. This result indicates that serum albumin and $\gamma$ globulin are selectively adsorbed on the hydrophilic and hydrophobic domains.

Figure 5 shows the micrographs of the surface of the block copolymer containing 0.884-mol fraction of HEMA before and after adsorbing serum albumin and $\gamma$-globulin. In the case of the block copolymer which has 0.884-mol fraction of HEMA, the hydrophobic domains were dispersed as island state in the sea of the hydrophilic region. The white spots of the hydrophobic domains remained in the albumin system as shown in Figure 5B. In the system of $\gamma$-globulin, the dark areas showed adsorbed $\gamma$-globulin (Figure 5C), because only $\gamma$-globulin was stained by the short time exposure to osmium tetroxide, in the same manner as for the blendmer system.

That is, Figures 3, 4, and 5 show that the adsorption of serum albumin might be specific to the hydrophilic domains, while $\gamma$-globulin is ad-

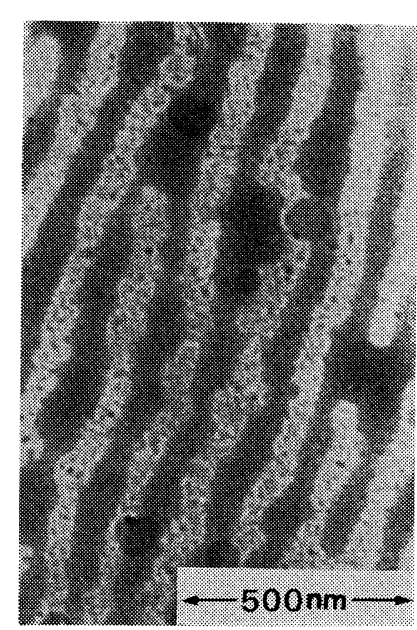

A

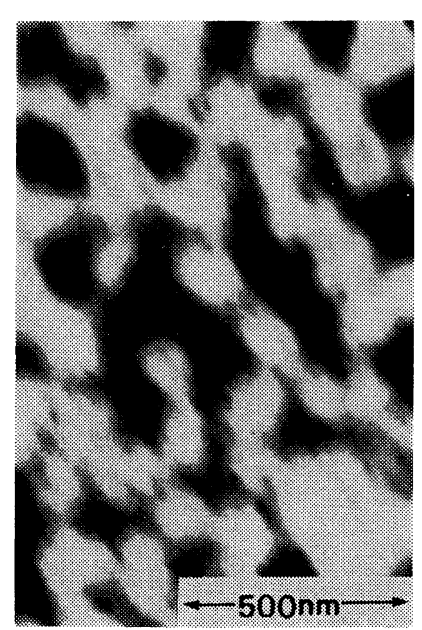

B

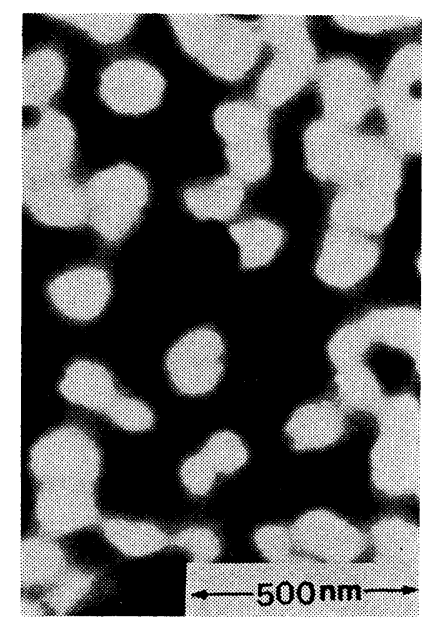

C

Figure 4. Adsorption of plasma protein on the surface of HEMA-styrene ABA-type block copolymer containing $0.608-\mathrm{mol}$ fraction of HEMA: (A) the surface of the block copolymer stained with osmium tetroxide; (B) the surface after adsorbing bovine serum albumin, stained with osmium tetroxide; (C) the surface after adsorbing $\gamma$-globulin, stained with osmium tetroxide. 


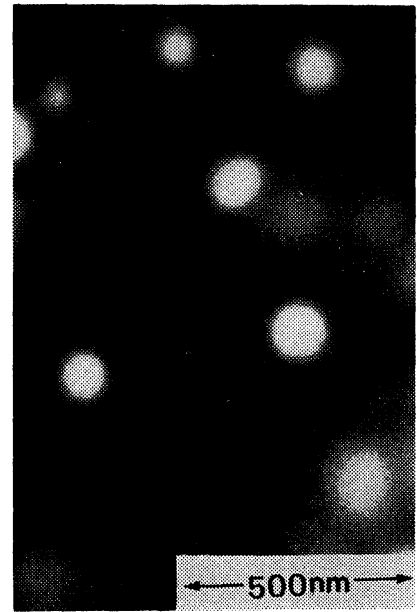

A

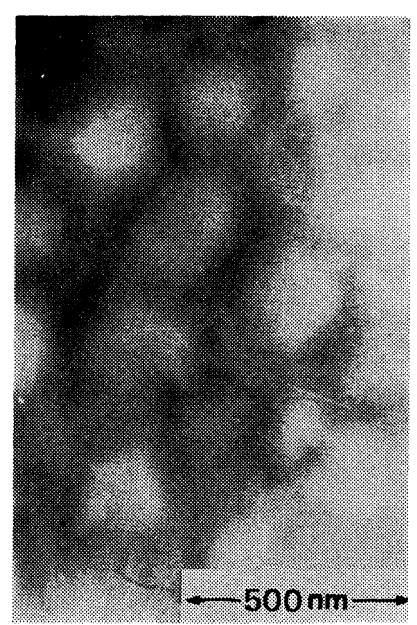

B

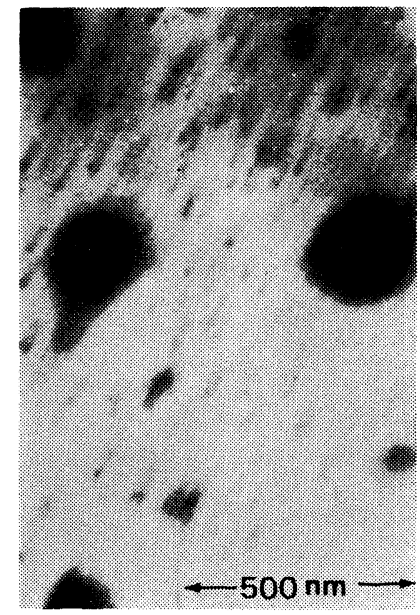

$\mathrm{C}$

Figure 5. Adsorption of plasma protein on the surface of HEMA-styrene ABA-type block copolymer containing $0.884-\mathrm{mol}$ fraction of HEMA: (A) the surface of the block copolymer stained with osmium tetroxide; (B) the surface after adsorbing bovine serum albumin, stained with osmium tetroxide; $(C)$ the surface after adsorbing $\gamma$-globulin, stained with osmium tetroxide.

sorbed selectively on the hydrophobic domains. The selectivity of the adsorption of protein by the styrene rich block copolymer (Figure 3) seems to be a little higher than that by HEMA rich copolymers (Figures 4 and 5). The wettability of the block copolymer of HEMA mole fraction 0.347 was lower than the HEMA rich copolymers. ${ }^{4}$ It may be suggested that the adsorption and spreading of the plasma proteins in the liquid-solid interface was influenced by the wettability of the polymer surface, which controlled the selective adsorption of the proteins on the microstructure of the copolymer.

As is remarked above, the adsorption behavior was examined from the viewpoint of the specificity of plasma protein species. Other important factors, i.e., the quantity and conformation of the adsorbed plasma proteins, should be analyzed by means of circular dichroism, ultraviolet, and Fourier transformation infrared spectroscopies, ${ }^{6}$ for example.

\section{CONCLUSION}

Serum albumin is adsorbed on the hydrophilic domain and avoids the hydrophobic domain, while $\gamma$-globulin is selectively adsorbed on the hydrophobic domains, as shown in the schematic representation of Figure 6 . The selective adsorption of serum albumin and $\gamma$-globulin on the surfaces having the hydrophilic and the hydrophobic microdomains was distinguished directly by means of
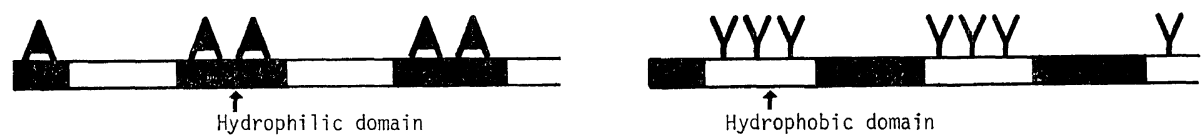

Figure 6. Adsorption of plasma protein on the surface of polymer which has a microphase separated structure composed of hydrophilic and hydrophobic micro-domains. 
electron microscopic observations using the osmium tetroxide fixation technique.

\section{REFERENCES}

1. D. J. Lyman, K. Knutson, B. McNeill, and K. Shibatani, Trans. Am. Soc. Artif. Int. Organs, 21, 49 (1972); S. W. Kim, R. G. Lee, H. Oster, D. Coleman, J. D. Andrade, D. J. Lentz, and D. Olsen, ibid., 2, 449 (1974); M. B. Zucker, and L. Vroman, Proc. Soc. Exp. Biol. Med., 131, 318 (1969).

2. Y. Imai, Kobunshi (High Polymers, Japan), 21, 569 (1972).
3. P. N. Sawyer, C. Burrowes, J. Ogoniak, A. O. Smith, and S. A. Wesolowski, Trans. Am. Soc. Artif. Int. Organs, 10, 316 (1964).

4. T. Okano, M. Katayama, and I. Shinohara, $J$. Appl. Polym. Sci., in press; T. Okano, M. Katayama, S. Mogi, and I. Shinohara, Nippon Kagakukaishi (J. Chem. Soc. Japan, Chem. Ind. Chem.), 1977 (1), 88 (1977).

5. T. Okano, M. Katayama, and I. Shinohara, ibid., 1976 (12), 1908 (1976).

6. Y. Sakurai and T. Akaike, IUPAC Post-Congress Symposium in Kyoto, Session IIA Biomedical Materials, 14 (1977). 